\title{
COVID-CT-Mask-Net: Prediction of COVID-19 from CT Scans Using Regional Features
}

\author{
Aram Ter-Sarkisov \\ CitAI, Artificial Intelligence Research Centre \\ Department of Computer Science \\ City, University of London
}

\begin{abstract}
We present COVID-CT-Mask-Net model that predicts COVID-19 from CT scans. The model works in two stages: first, it detects the instances of ground glass opacity and consolidation in CT scans, then predicts the condition from the ranked bounding box detections. To develop the solution for the three-class problem (COVID, common pneumonia and control), we used the COVIDx-CT dataset derived from the dataset of CT scans collected by China National Center for Bioinformation. We use about $5 \%$ of the training split of COVIDx-CT to train the model, and without any complicated data normalization, balancing and regularization, and training only a small fraction of the model's parameters, we achieve a $\mathbf{9 0 . 8 0} \%$ COVID sensitivity, $\mathbf{9 1 . 6 2} \%$ common pneumonia sensitivity and $\mathbf{9 2 . 1 0} \%$ normal sensitivity, and an overall accuracy of $\mathbf{9 1 . 6 6} \%$ on the test data (21182 images), bringing the ratio of test/train data to $\mathbf{7 . 0 6}$, which implies a very high capacity of the model to generalize to new data. We also establish an important result, that ranked regional predictions (bounding boxes with scores) in Mask R-CNN can be used to make accurate predictions of the image class. The full source code, models and pretrained weights are available on https://github.com/AlexTS1980/COVID-CT-Mask-Net.
\end{abstract}

\section{Introduction}

Deep learning COVID diagnostic tools for a 3 classes problem (COVID vs common pneumonia vs control) from CT images include COVIDNet-CT [GWW20], that consists of a single feature extractor trained on COVIDx-CT dataset split, COVNet (augmented ResNet50) [LQX ${ }^{+}$20] and ResNet18 [BGCB20]. Distinguishing between 3 classes is a more challenging problem than COVID vs Pneumonia or COVID vs Control, due to the larger number of potential false predictions. In order to achieve the state-of-the-art [GWW20] accuracy, large amounts of data are required to train (about $60 \mathrm{~K}$ images) the model, that are often not available, which explains demand for various augmentations.

One approach that some publications use for COVID prediction, is the semantic segmentation model, e.g. U-Net in $\left[\mathrm{ZLS}^{+} 20, \mathrm{WGM}^{+}\right.$20] as a pre-processing step: its output (mask) is used by the classifier to enhance the prediction. The advantage of using a segmentation model is that it is capable of explicitly learning and predicting areas of infection associated with COVID. For a binary classification problem (COVID vs control, COVID vs common pneumonia), COVID-CT [ZZHX20] and JCS (Joint Classification and Segmentation) [WGM ${ }^{+} 20$ ] are publicly available. COVID-CT combines lung masks predicted using U-Net with deep image features extracted using DenseNet169 and ResNet50 to predict the class, achieving an overall accuracy of $89 \%$ on the test data of about 350 images. JCS uses a similar approach, but with additional loss functions at deep layers (multiscale training), achieving a Dice score of 0.783 on the test data of about $120 \mathrm{~K}$ images.

A number of reviews have compared different feature extractors and models directly to establish the best one for accuracy: $\left[\mathrm{SZL}^{+}, \mathrm{SWS}^{+} 20, \mathrm{LYZ}^{+}\right.$20]. From these papers it seems that for CT scans data, ResNet50 (+Feature Pyramid 
Network), ResNeXt and DenseNet121 produce the highest overall accuracy. At least one recent publication [ARK20] discusses the use of Mask R-CNN for predicting COVID from the segmentation of CT scans.

The majority of COVID deep learning models use radiography (X-rays) data due to its prevalence, e.g. the stateof-the-art COVID-Net [WW20] that has an architecture similar to COVIDNet-CT. To the best of our knowledge, only COVIDNet-CT, COVNet and ResNet18 [BGCB20] use CT scans for a 3-class (COVID vs common pneumonia vs control) rather than a binary (COVID vs control) problem. This problem is more challenging and realistic, both due to the fact that COVID and common pneumonia symptoms are similar in many ways, on CT scans they manifest in a different way $\left[Z Z X^{+} 20 a, Z Z X^{+} 20 b, Y^{+} R^{+} 20\right]$, but these differences are subtle. These models have the following drawbacks: COVIDNet-CT requires a large training data with various augmentations and class balancing to achieve the state-of-theart accuracy and COVID sensitivity, COVNet was evaluated on a small dataset (about 500 images), ResNet18 [BGCB20] is not publicly available, it has a low COVID sensitivity (81.2\%) and was evaluated on a small data (90 images).

In this paper we would like to address these shortcomings by extending the semantic segmentation+classification solution to instance segmentation+classification using Mask R-CNN. Mask R-CNN [HGDG17] and Faster R-CNN [RHGS15] are the state-of-the-art models in instance segmentation and object detection. Mask R-CNN is an extension of Faster R-CNN with a mask prediction branch at an instance level. This is different to semantic segmentation models like Fully Convolutional Network (FCN) [LSD15] and U-Net [RFB15], which predict objects at pixel level. Mask R-CNN differentiates between different instances belonging to the same class by predicting their location (bounding box coordinates) using Region Proposal Network (RPN) and Regions of Interest (RoI). Each predicted object has therefore three features: bounding box, class and mask. The strength of Faster/Mask R-CNN comes from the fact that the model constructs samples of data from each image (regional features) to make predictions about the instances. This leverages the scarcity of the training data, and we use this strength both to obtain accurate predictions and use a small sample for training. By augmenting Mask R-CNN with a classification module, we extend its ability to detect objects to making prediction about the whole image.

The novelty of our approach to COVID-19 prediction can be summarized in the following way:

1. Solution: we use approx. $5 \%$ of the COVIDx-CT training data, and $3 \%$ of the total data, and, without any data augmentation, e.g. class weights, background removal and batch balancing, on which COVIDNet-CT depends, achieve $90.80 \%$ COVID sensitivity, and $91.66 \%$ overall accuracy on the full test split (21182 images).

2. Architecture: we repurpose Mask R-CNN to predict the class of the image using bounding box predictions by leveraging the ability of Mask R-CNN to extract regions of interest (RoIs) from deep features and obtain spatial predictions (bounding boxes) from them to construct a batch of ranked regional predictions in each image and use it to predict the global (image) class.

3. We solve both segmentation and prediction problems by training two models. Mask R-CNN segmentation model predicts and segments the cases of Ground Glass Opacity and Consolidation in CT scans, COVID-CT-Mask-Net uses this model to predict the class of the image.

Overall, we use much less training data than COVIDNet-CT, achieve a better overall accuracy and COVID sensitivity than COVNet[ZZX ${ }^{+}$20a] and ResNet18[BGCB20], and our solution has a better potential for generalization to other datasets.

\section{Data}

\subsection{Segmentation}

For our segmentation model we use the publicly available dataset published by China National Center for Bioinformation [ZLS $\left.{ }^{+} 20\right]$ consisting of 650 scans across 150 patients with various stages of COVID. A total of $1+2$ classes are segmented 

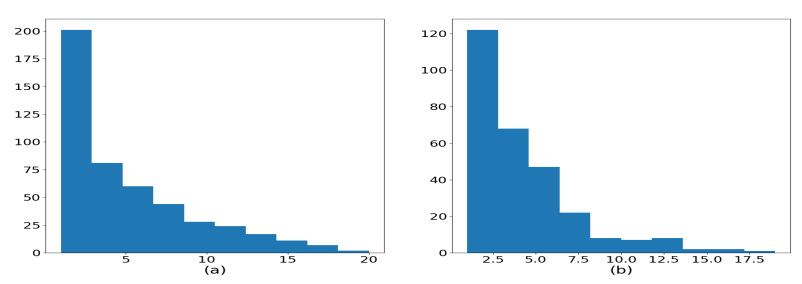

(1) Number of instances/image
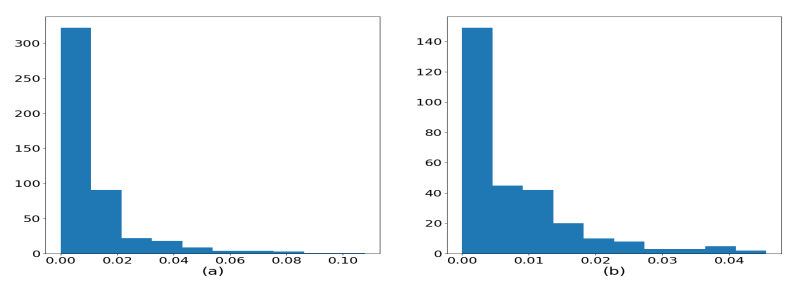

(2) Ratio of the total area of instances to the image size

Figure 1: Distribution of the COVID correlates in the segmentation data. Column (a): Ground Glass Opacity, Column (b): Consolidation. In 1.1, we plotted the number of separate GGO and C occurrences in each image. The absolute majority of images have a small number ( $<5$ occurrences of each type). Histograms in Figure 1.2 complements this finding with the area of occurrences: the absolute majority of them are very small: GGO are $<2 \%$ of the image size and C are $<1 \%$. This means that the absolute majority of CT scans has a small number of small occurrences.

\begin{tabular}{|l|c|c|c|c|c|c|}
\hline Model & $\begin{array}{c}\text { Total } \\
\text { \#parameters }\end{array}$ & $\begin{array}{c}\text { \#Trainable } \\
\text { parameters }\end{array}$ & Training & Validation & Test & $\begin{array}{c}\text { Ratio } \\
\text { Test/Train }\end{array}$ \\
\hline Mask R-CNN (segmentation) & $31.78 \mathrm{M}$ & $31.78 \mathrm{M}$ & 600 & 150 & - & - \\
\hline COVID-CT-Mask-Net (heads) & & $2.25 \mathrm{M}$ & & & & \\
COVID-CT-Mask-Net (heads+BN) & $34.14 \mathrm{M}$ & $2.36 \mathrm{M}$ & $3 \mathrm{~K}$ & $20.6 \mathrm{~K}$ & $21.1 \mathrm{~K}$ & 7.06 \\
COVID-CT-Mask-Net (full) & & $34.14 \mathrm{M}$ & & & & \\
\hline COVIDNet-CT [GWW20] & $1.8 \mathrm{M}$ & $1.8 \mathrm{M}$ & $60 \mathrm{~K}$ & $20.6 \mathrm{~K}$ & $21.1 \mathrm{~K}$ & 0.353 \\
COVNet [LQX+20] & $25.61 \mathrm{M}$ & $25.61 \mathrm{M}$ & $3 \mathrm{~K}$ & 370 & 438 & 0.129 \\
ResNet18 [BGCB20] & $11.69 \mathrm{M}$ & $11.69 \mathrm{M}$ & & 528 & 90 & 0.17 \\
\hline
\end{tabular}

Table 1: Comparison of models' sizes and data splits used to training, validation and testing.

at pixel level: lung field (normal), which we merged with the background, ground glass opacity (GGO) and consolidation (C). These two conditions are often associated with various stages of COVID and other viral diseases, so we treat them as positive classes. We randomly split the provided dataset into 500 training and 150 validation images, maintaining the patient consistency, therefore some slices of COVID-positive patients do not contain positive classes. The challenge of the data is summarized in Figure 1: it is clear that positive scans can contain a small number of small objects of either class, and overall, the proportion of positive areas is very low, making the problem of segmenting them a serious challenge.

\subsection{Classification}

To compare our model to COVIDNet-CT we used the dataset labelled at the image level provided by the same source, [ZLS $\left.{ }^{+} 20\right]$, http://ncov-ai . big.ac.cn/download and the split, COVIDx-CT that was used to train COVIDNet-CT model (https://github.com/haydengunraj/COVIDNet-CT), which is publicly available. In total 104900 images from the CNCB dataset were partitioned into $60 \%$ training, $20 \%$ validation and $20 \%$ test data. The difference between COVIDx-CT and the source data is that for COVID and pneumonia classes, only scans with observable infected regions were selected [GWW20].

One of the advantages of our model is a small dataset used for training. We extracted randomly 3000 images from COVIDx-CT training data (1000/class), while maintaining the full size of the validation (21036 images) and test (21182 images) for direct comparison. In the validation split, the shares or Normal/Pneumonia/COVID classes are $43 \% / 35 \% / 22 \%$, in the test split they are $45 \% / 35 \% / 20 \%$. 


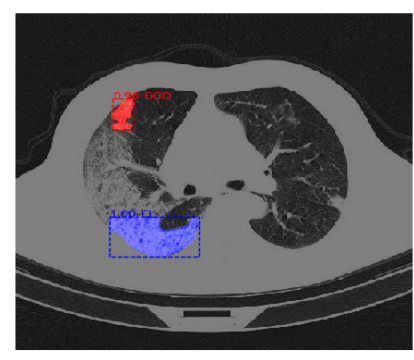

a

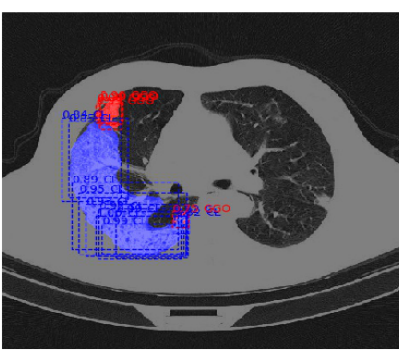

b

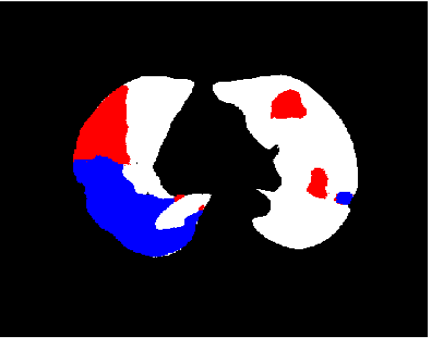

c

(1) Output of the segmentation model for a lung slice with both Ground Glass opacity and Consolidation

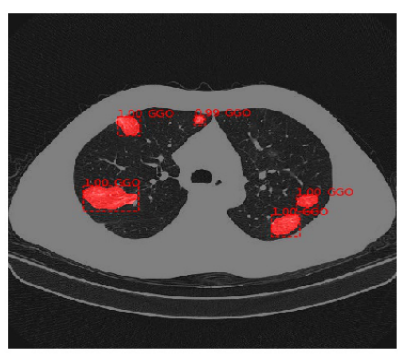

a

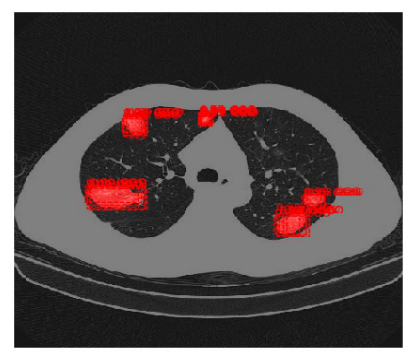

b

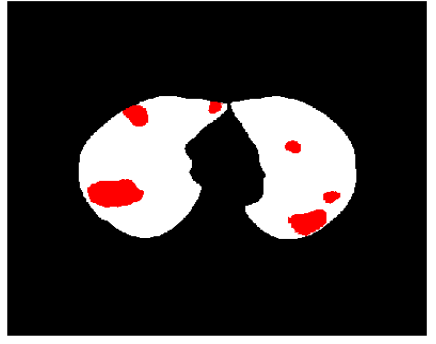

C

- GroundGlassopacity Consolidation

(2) Output of the segmentation model for a lung slice with only Ground Glass Opacity

Figure 2: Ground Glass Opacity and Consolidation segmentation predicted by the same model with RoI Non-Max Suppression Threshold $=0.25$ in column (a) and RoI Non-Max Suppression Threshold $=0.75$ in column (b). Column (c) is the ground-truth mask. Predictions with scores above RoI score $e_{\theta}=0.75$ for each detection and all pixel-level mask logits $>0$ are considered positive.

\section{Our Approach}

Our solution is split into two stages: first, we train an instance segmentation model (Figure 3.1) to predict masks of GGO and $C$ areas. After validation, this model is augmented with a classification module $S$ (Figure 3.3) that uses ranked bounding box predictions to classify the whole input image (Figure 3.2).

\subsection{Segmentation model}

Faster R-CNN[RHGS15] and Mask R-CNN [HGDG17] extract regional features from one of the backbone feature maps in three steps: 1) align the coordinates predicted by RPN to the feature map, 2) crop them, 3) resize (RoI align) to the predefined size. As a result, all RoIs are of the same size: $C \times H \times W(C$ : number of maps, $H, W$ : height and width of the map). A sample of positive and negative RoIs is constructed to predict the class and refined coordinates of the object. The object's mask is predicted independently of other objects and classes, by comparing the mask's logits to the ground truth mask. We train Mask R-CNN to construct the model that is capable of finding a number of small objects of varying shapes, which are widespread in CT scans of patients with COVID, see Figure 1. Most anchor sizes are small $(<32 \times 32$ pixels) and have a large number of scales (6 in total between 0.1 and 2), allowing for accurate detection of various shapes of GGO and C. For the explantion of the details of the model's hyperparameters (Non-max suppression, RPN/RoI batch size, foreground and background selection thresholds, etc) see [HGDG17] and our implementation. Examples of segmentation model's outputs are presented in Figure 2. We use Torchvision implementation of Mask 


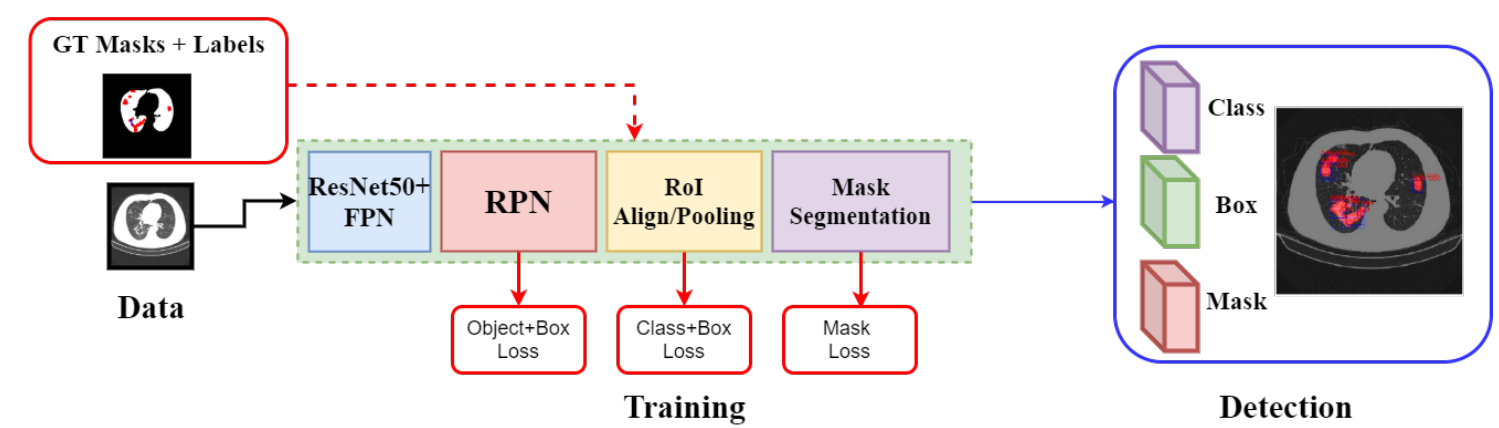

(1) Mask R-CNN model. Black line is the data input. Labels are the class of the positive objects and bounding box coordinates and binary masks for each instance. Broken red line are the labels and gt masks during training. Red lines are the losses computer during training. Blue line is the output during inference: class, box and mask predictions. The output image is overlaid with the best predictions

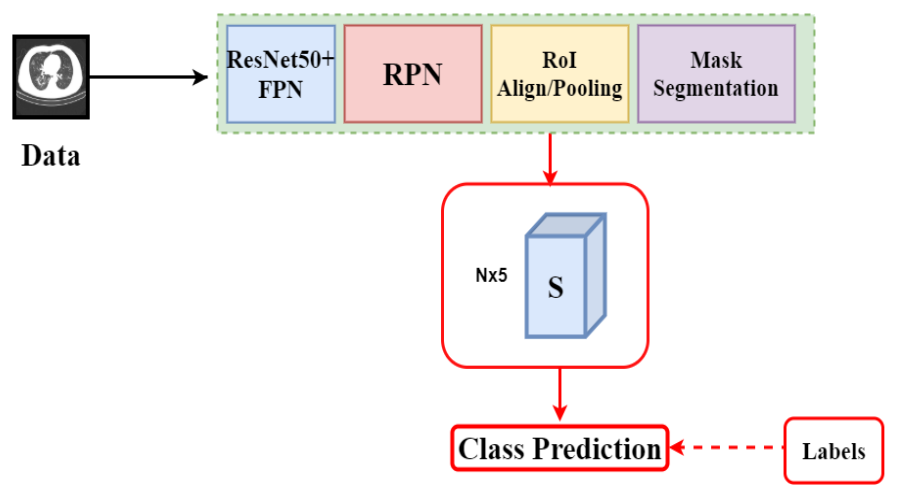

(2) COVID-CT-Mask-Net. RPN and RoI do not compute any losses. The new classification module S (Figure 3.3) take the batch size $N$ of the ranked encoded boxes with their scores as an input and predicts the class of the input image.

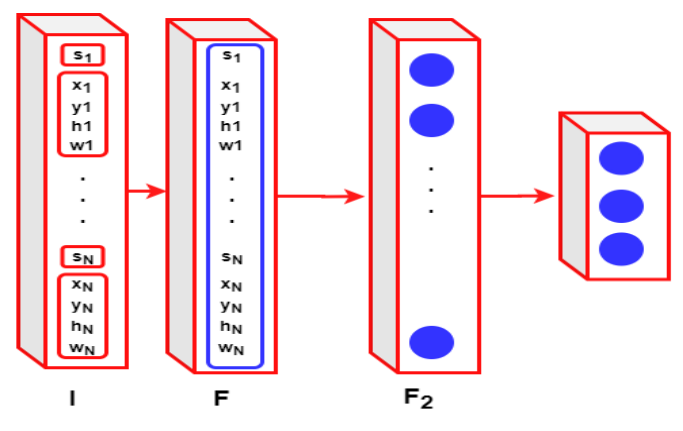

(3) Classification module $S$ of COVID-CT-Mask-Net: The input $I$ is resized from $N \times 5$ to feature vector $F$ size $1 \times N \cdot 5$. Fully connected layer $F_{2}$ is size 1024, and the last prediction layer outpus 3 logit (scores) , 1 per image class.

Figure 3: Architecture of the segmentation model, COVID-CT-Mask-Net and classification module $S$. 


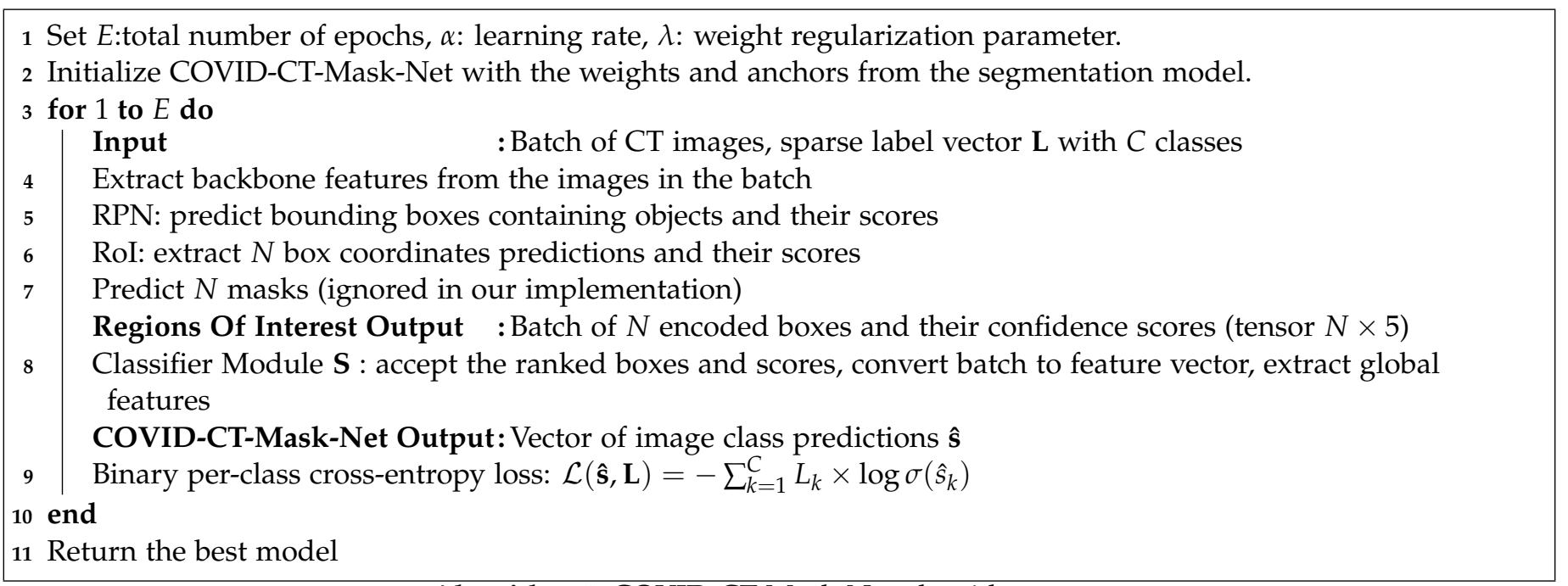

Algorithm 1: COVID-CT-Mask-Net algorithm.

R-CNN https://github.com/pytorch/vision/tree/master/torchvision/models/detection with 5 loss functions: binary cross-entropy for class and Smooth1Loss for bounding box coordinates in RPN, multilabel cross-entropy for class and Smooth1Loss for bounding box coordinates in RoI and pixel-wise class-conditional binary cross-entropy for masks.

\subsection{COVID-CT-Mask-Net}

We augment Mask R-CNN with a classification module $S$ that makes predictions about the whole image. Details of the COVID-CT-Mask-Net algorithm are presented in Algorithm 1 and Figure 3.2. The details of module $S$ are in Figure 3.3.

Batch to features One of the most important steps in Mask R-CNN is construction of the batch in the image by taking a sample of positive (score $>\theta_{\text {pos }}$ ) and negative (score $<\theta_{\text {neg }}$ ) RoIs at the training stage. At the inference stage, each RoI predicts a number of encoded bounding boxes (one per class), each with a confidence score, from which, after discarding predictions with scores below score ${ }_{\theta}$, and overlapping predictions, a batch of atmost $N$ highest-scoring bounding box predictions is extracted. Mask R-CNN encodes coordinates to make predictions independent of the images size, so this is a form of normalization.

We transform this process for the purpose of whole image classification, as we need low-scoring regions too, to give the classifier sufficient information, especially for negative images without any GGO and C conditions. To obtain a fixed-size output from RoI stage, we set the score $\theta=-0.01$, so that even very low-scoring predictions are accepted, and RoI output size is fixed to $N \times 5$ ( $N$ encoded bounding box coordinates+confidence score). Normally, RoI decodes these bounding box coordinates by scaling them to the size of the input image, and ranks them based on the confidence score. We ignore the scaling to the image size and use these ranked encoded coordinates with their score as an input in the classification module $S$. The advantage of this approach is that, even if the highest score is very low (in negative images), the predicted coordinates are still ranked (highest to lowest). This ranking pattern is something the classifier $S$ can learn. $S$ resizes this input into a single feature vector size $1 \times(N \cdot 5)$, which maintains the rank of detections. After some filtering and feature extraction, the module predicts the scores for the whole image (COVID, pneumonia, control), see Figure 3.3.

Non-maximum suppressions (NMS) NMS is the threshold value for discarding predictions of the object of the same class. Setting it high means allowing a larger number of predictions in the training sample with IoU> pre-defined NMS threshold. We established that the model learns that overlapping (adjacent) regions with high scores are associated with 


\begin{tabular}{|c|c|c|c|c|}
\hline Model & COVID & Pneumonia & Normal & Overall \\
\hline COVID-CT-Mask-Net(heads) & $76.30 \%(81.13 \%)$ & $71.13 \%(67.70 \%)$ & $82.37 \%(83.38 \%)$ & $77.20 \%$ \\
COVID-CT-Mask-Net(heads+BN) & $\mathbf{9 0 . 8 0} \%(\mathbf{9 4 . 7 5} \%)$ & $91.62 \%(87.08 \%)$ & $91.10 \%(94.33 \%)$ & $91.66 \%$ \\
COVID-CT-Mask-Net(full) & $82.26 \%(87.01 \%)$ & $\mathbf{9 1 . 7 0} \%(\mathbf{9 5 . 2 2} \%)$ & $\mathbf{9 7 . 2 1} \%(\mathbf{9 5 . 3 3} \%)$ & $\mathbf{9 2 . 2 2} \%$ \\
\hline
\end{tabular}

Table 2: Sensitivity (precision) and overall accuracy results on COVIDx-CT test data (21182 images)

higher probability of presence of COVID, and hence it improves sensitivity at the cost of lower overall accuracy. To over come this fact, since in many scans GGO or C areas can be very small, and hence produce only one or very few high-scoring box predictions, we set the NMS threshold to 0.75 in both models, thus increasing the sensitivity to COVID.

\section{Experiments}

For COVID-CT-Mask-Net, we re-implement Torchvision's Mask R-CNN library. During the training of the classifier, RPN and RoI do not compute any loss. The object threshold RoI score $\theta_{\theta}$ is set to -0.01 to accept all box predictions, even with low scores, to guarantee the batch size and the feature vector remain the same in S. We train COVID-CT-Mask-Net in three different ways (Table 1): only classification module $S$, module $S+$ batch normalization layers, and full model. To train the full model, a large hack had to be applied both to the RPN and RoI modules: all layers in these modules were set to the training mode, the weights made trainable, and loss computation and all related sampling operations were switched off. Therefore, although formally Mask R-CNN layers were in the evaluation mode, in fact they were updated. Compared to other models, we use a small fraction of the dataset of COVIDx-CT for training, while maintaining the full size of the test and validation sets. As a result, the test/train splits ratio is 7.06, which is the new state-of-the-art, and demonstrates the ability of COVID-CT-Mask-Net to generalize to the unseen data. We use Adam optimizer, learning rate $1 e-5$, weight regularization parameter $1 e-3$, and train each algorithm for 50 epochs. For other details of the segmentation algorithm and COVID-CT-Mask-Net see our implementation, https ://github. com/AlexTS1980/COVID-CT-Mask-Net.

To evaluate each model, we compute the sensitivity/recall and precision/positive predictive value (PPV) for each class $C$ and the overall accuracy of the model:

$$
\begin{aligned}
\text { Sensitivity }(C) & =\frac{\operatorname{True} \operatorname{Positive}(C)}{\operatorname{True} \operatorname{Positive}(C)+\text { False Negative }(C)} \\
\text { Precision }(C) & =\frac{\operatorname{True} \operatorname{Positive}(C)}{\operatorname{True~Positive}(C)+\operatorname{False} \operatorname{Positive}(C)} \\
\text { Overall Accuracy } & =\frac{\sum_{C} \operatorname{True} \operatorname{Positive}(C)}{\sum_{C} \text { True Positive }(C)+\sum_{C} \operatorname{False} \operatorname{Negative}(C)}=\frac{\sum_{C} \operatorname{True} \operatorname{Positive}(C)}{\sum_{C} \operatorname{True} \operatorname{Positive}(C)+\sum_{C} \text { False Positive }(C)}
\end{aligned}
$$

Best results for each trained version of COVID-CT-Mask-Net are presented in Table 2. The model with the classifier head + batch normalization layers produces precision $>90 \%$ across all classes. Comparison of our results to other COVID CT detectors for 3 classes is presented in Tables 1 and 3. For COVIDNet-CT we used the best reported model (COVIDNet-CT-A), COVNet and [BGCB20] report only one model.

\section{Conclusions}

It is often a challenge to find a sufficiently large dataset to train models for accurate predictions of COVID. One of the strongest features of COVID-CT-Mask-Net's methodology is the ability to train on very small amounts of data without any balancing and augmentation tweaks. We trained our model on less than 5\% of COVIDx-CT training split, and evaluated it on more than 21000 test images achieving a $91.66 \%$ overall accuracy and $90.80 \%$ COVID sensitivity. The 


\begin{tabular}{|c|c|c|c|}
\hline Model & COVID Sensitivity & Overall accuracy & COVID prevalence \\
\hline Ours & $90.80 \%$ & $91.66 \%$ & $20 \%$ \\
\hline COVIDNet-CT [GWW20] & $92.49 \%$ & $97.57 \%$ & $20 \%$ \\
COVNet [LQX ${ }^{+}$20] & $90.00 \%$ & $89.04 \%$ & $30 \%$ \\
ResNet18 [BGCB20] & $81.30 \%$ & $86.70 \%$ & $35.79 \%$ \\
\hline
\end{tabular}

Table 3: Comparison to other models. The results for COVIDNet-CT were obtained by running the publicly available model (https://github.com/haydengunraj/COVIDNet-CT) on the same test split, results for the other two models are taken from the publication. Last column is the share of COVID observations in the test split. Test split for COVNet has 438 images, ResNet18 90 images.

model can be easily and quickly finetuned to new CT data to achieve high COVID detection rate. The source code with all models and weights are on https://github.com/AlexTS1980/COVID-CT-Mask-Net.

\section{References}

[ARK20] Muhammad Aleem, Rahul Raj, and Arshad Khan. Comparative performance analysis of the resnet backbones of mask rcnn to segment the signs of covid-19 in chest ct scans. arXiv preprint arXiv:2008.09713, 2020.

[BGCB20] Charmaine Butt, Jagpal Gill, David Chun, and Benson A Babu. Deep learning system to screen coronavirus disease 2019 pneumonia. Applied Intelligence, pages 1-7, 2020.

[GWW20] Hayden Gunraj, Linda Wang, and Alexander Wong. Covidnet-ct: A tailored deep convolutional neural network design for detection of covid-19 cases from chest ct images. arXiv preprint arXiv:2009.05383, 2020.

[HGDG17] Kaiming He, Georgia Gkioxari, Piotr Dollár, and Ross Girshick. Mask r-cnn. In Proceedings of the IEEE international conference on computer vision, pages 2961-2969, 2017.

$\left[\mathrm{LQX}^{+} 20\right]$ Lin Li, Lixin Qin, Zeguo Xu, Youbing Yin, Xin Wang, Bin Kong, Junjie Bai, Yi Lu, Zhenghan Fang, Qi Song, et al. Artificial intelligence distinguishes covid-19 from community acquired pneumonia on chest ct. Radiology, 2020.

[LSD15] Jonathan Long, Evan Shelhamer, and Trevor Darrell. Fully convolutional networks for semantic segmentation. In Proceedings of the IEEE conference on computer vision and pattern recognition, pages 3431-3440, 2015.

[LYZ 20] Boyi Liu, Bingjie Yan, Yize Zhou, Yifan Yang, and Yixian Zhang. Experiments of federated learning for covid-19 chest X-ray images. arXiv preprint arXiv:2007.05592, 2020.

[RFB15] Olaf Ronneberger, Philipp Fischer, and Thomas Brox. U-net: Convolutional networks for biomedical image segmentation. In International Conference on Medical image computing and computer-assisted intervention, pages 234-241. Springer, 2015.

[RHGS15] Shaoqing Ren, Kaiming He, Ross Girshick, and Jian Sun. Faster r-cnn: Towards real-time object detection with region proposal networks. In Advances in neural information processing systems, pages 91-99, 2015.

[SWS ${ }^{+}$20] Feng Shi, Jun Wang, Jun Shi, Ziyan Wu, Qian Wang, Zhenyu Tang, Kelei He, Yinghuan Shi, and Dinggang Shen. Review of artificial intelligence techniques in imaging data acquisition, segmentation and diagnosis for covid-19. IEEE reviews in biomedical engineering, 2020.

[SZL $\left.{ }^{+}\right] \quad Y i n g$ Song, Shuangjia Zheng, Liang Li, Xiang Zhang, Xiaodong Zhang, Ziwang Huang, Jianwen Chen, Huiying Zhao, Yusheng Jie, Ruixuan Wang, Yutian Chong, Jun Shen, Yunfei Zha, and Yuedong Yang. Deep learning enables accurate diagnosis of novel coronavirus (covid-19) with ct images. medRxiv. 
medRxiv preprint doi: https://doi.org/10.1101/2020.10.11.20211052; this version posted November 9, 2020. The copyright holder for this preprint

(which was not certified by peer review) is the author/funder, who has granted medRxiv a license to display the preprint in perpetuity.

It is made available under a CC-BY 4.0 International license .

$\left[\mathrm{WGM}^{+}\right.$20] Yu-Huan Wu, Shang-Hua Gao, Jie Mei, Jun Xu, Deng-Ping Fan, Chao-Wei Zhao, and Ming-Ming Cheng. Jcs: An explainable covid-19 diagnosis system by joint classification and segmentation. arXiv preprint arXiv:2004.07054, 2020.

[WW20] Linda Wang and Alexander Wong. Covid-net: A tailored deep convolutional neural network design for detection of covid-19 cases from chest x-ray images. arXiv preprint arXiv:2003.09871, 2020.

[YWR $\left.{ }^{+} 20\right]$ Tao Yan, Pak Kin Wong, Hao Ren, Huaqiao Wang, Jiangtao Wang, and Yang Li. Automatic distinction between covid-19 and common pneumonia using multi-scale convolutional neural network on chest ct scans. Chaos, Solitons \& Fractals, 140:110153, 2020.

[ZLS ${ }^{+}$20] Kang Zhang, Xiaohong Liu, Jun Shen, Zhihuan Li, Ye Sang, Xingwang Wu, Yunfei Zha, Wenhua Liang, Chengdi Wang, Ke Wang, et al. Clinically applicable ai system for accurate diagnosis, quantitative measurements, and prognosis of covid-19 pneumonia using computed tomography. Cell, 2020.

[ZZHX20] Jinyu Zhao, Yichen Zhang, Xuehai He, and Pengtao Xie. Covid-ct-dataset: a ct scan dataset about covid-19. arXiv preprint arXiv:2003.13865, 2020.

[ZZX+20a] Wei Zhao, Zheng Zhong, Xingzhi Xie, Qizhi Yu, and Jun Liu. Ct scans of patients with 2019 novel coronavirus (covid-19) pneumonia. Theranostics, 10(10):4606, 2020.

$\left[Z_{Z}{ }^{+}\right.$20b] Wei Zhao, Zheng Zhong, Xingzhi Xie, Qizhi Yu, and Jun Liu. Relation between chest ct findings and clinical conditions of coronavirus disease (covid-19) pneumonia: a multicenter study. American Journal of Roentgenology, 214(5):1072-1077, 2020. 